\title{
ON A FIRST PASSAGE PROBLEM FOR BRANCHING BROWNIAN MOTIONS
}

\author{
By Ingemar KaJ ${ }^{1}$ and PaAvo Salminen ${ }^{2}$ \\ Uppsala Universitet and Åbo Akademi
}

\begin{abstract}
Consider a (space-time) realization $\omega$ of a critical or subcritical onedimensional branching Brownian motion. Let $Z_{x}(\omega)$ for $x \geq 0$ be the number of particles which are located for the first time on the vertical line through $(x, 0)$ and which do not have an ancestor on this line. In this note we study the process $Z=\left\{Z_{x} ; x \geq 0\right\}$. We show that $Z$ is a continuous-time Galton-Watson process and compute its creation rate and offspring distribution. Here we use ideas of Neveu, who considered a similar problem in a supercritical case. Moreover, in the critical case we characterize the continuous state branching processes obtained as weak limits of the processes $Z$ under rescaling.
\end{abstract}

1. Introduction and basic definitions. Let $X=\left\{X_{t} ; t \geq 0\right\}, X_{0}=x$, be a branching Brownian motion in $\mathbf{R}$ with a constant creation rate $\alpha$ and the offspring distribution $p=\left\{p_{k} ; k=0,1,2, \ldots\right\}$. It is assumed that $p_{1}<1$ and that $X$ is (sub)critical, that is, $\sum_{k=0}^{\infty} k p_{k} \leq 1$. The canonical sample space of $X$ is a space of marked trees which we now describe.

Consider the set

$$
U:=\bigcup_{n=1}^{\infty} \mathbf{N}_{+}^{n} \cup\{0\}, \quad \mathbf{N}_{+}:=\{1,2, \ldots\}
$$

and let $\omega$ be a subset of $U$ with the properties (cf. Neveu [9]):

1. $0 \in \omega$,

2. $\forall u, v \in U: u v \in \omega \Rightarrow u \in \omega$,

3. $\forall u \in \omega \exists \nu^{u}(\omega) \in \mathbf{N} \forall j \in \mathbf{N}_{+}: u j \in \omega \Rightarrow 1 \leq j \leq \nu^{u}(\omega)$.

Such subsets $\omega$ are called trees and we denote the space of all trees by $\Omega$. Elements in $U$ are called particles. To explain the notation $u v$ in (2), let $u=\left(i_{1}, \ldots, i_{s}\right) \in U, v=\left(j_{1}, \ldots, j_{r}\right) \in U$, then $u v=\left(i_{1}, \ldots, i_{s}, j_{1}, \ldots, j_{r}\right) \in$ $U$. The variable $\nu^{u}$ in (3) gives the number of descendants of the particle $u$. A particle $v$ is called an ancestor of a particle $u$, denoted $v \leq u$, if there exists $w \in U$ such that $u=v w$. Defining $0 u=u$ and $u 0=u$, it is seen that for every $u \in U$ we have $u \leq u$ and $0 \leq u$.

\footnotetext{
Received April 1991; revised March 1992.

${ }^{1}$ Supported by a Swedish Natural Sciences Research Council postdoctoral fellowship.

, ${ }^{2}$ Supported in part by Magnus Ehrnrooths Foundation and the Academy of Finland. $A M S 1991$ subject classifications. 60J65, 60J80.

Key words and phrases. Branching Brownian motion, Galton-Watson process, Lévy process, super-Brownian motion, weak convergence, first passage.
} 
Let

$$
M:=\left\{(\zeta, \gamma) ; \zeta \in \mathbf{R}_{+}, \gamma:[0, \zeta) \rightarrow \mathbf{R}, \text { continuous, } \gamma(0)=0\right\}
$$

A marked tree $\omega^{0}$ (cf. Chauvin [3]) is defined as

$$
\omega^{0}:=\left(\omega,\left\{\left(\zeta^{u}, \gamma^{u}\right) ; u \in \omega\right\}\right),
$$

where $\omega \in \Omega$ and $\left(\zeta^{u}, \gamma^{u}\right) \in M$ for every $u \in \omega$. Let $\Omega^{0}$ denote the set of all marked trees. For a given $\omega^{0} \in \Omega^{0}$ let $\omega$ be the corresponding tree in (1.1). To emphasize the structure we often denote the marks in $\omega^{0}$ with $\left(\zeta^{u}\left(\omega^{0}\right), \gamma^{u}\left(\omega^{0}\right)\right)$.

The path of a particle $u \in \omega$ is defined as

$$
\xi_{t}^{u}\left(\omega^{0}\right)= \begin{cases}x+\gamma_{t}^{0}\left(\omega^{0}\right), & \text { if } u=0, t<\zeta^{0} \\ \xi_{\zeta^{v}-}^{v}\left(\omega^{0}\right)+\gamma_{t}^{u}\left(\omega^{0}\right), & \text { if } u \neq 0, t<\zeta^{u} \\ \Delta, & \text { if } t \geq \zeta^{u}\end{cases}
$$

where $u=v j$ for some $j, 1 \leq j \leq \nu^{v}(\omega)$, that is, $v$ is $u$ 's parent and $\Delta$ is a fictitious cemetery state. The parameter $t$ in (1.2), when $t<\zeta^{u}$, is called the age of the particle $u$.

Next we introduce some relevant $\sigma$-fields in $\Omega^{0}$ (cf. [3]). First, for every $u \in U$ define in the space $\Omega^{0, u}:=\left\{\omega^{0} ; u \in \omega\right\}$,

$$
\mathscr{F}_{t}^{u}:=\sigma\left\{\gamma_{s}^{u}\left(\omega^{0}\right) ; 0 \leq s<t \wedge \zeta^{u}\left(\omega^{0}\right), \omega^{0} \in \Omega^{0, u}\right\}
$$

and then, recursively,

$$
\begin{aligned}
& \mathscr{G}^{0}:=\left\{\varnothing, \Omega^{0}\right\}, \\
& \mathscr{G}^{u}:=\mathscr{G}^{v} \vee \mathscr{F}_{\infty}^{v} \wedge \sigma\left\{\nu^{v}(\omega) ; \omega^{0} \in \Omega^{0, v}\right\},
\end{aligned}
$$

where $u \neq 0$ and $v$ is $u$ 's parent. Intuitively, $\mathscr{G}^{u}$ contains information on the branch leading to the particle $u$. To include the history of the particle itself, set

$$
\begin{aligned}
\mathscr{H}_{t}^{u} & :=\mathscr{G}^{u} \vee \mathscr{F}_{t}^{u}, \\
\mathscr{H}_{\infty}^{u} & :=\bigvee_{t>0} \mathscr{H}_{t}^{u} \wedge \sigma\left\{\nu^{u}(\omega) ; \omega^{0} \in \Omega^{0, u}\right\}
\end{aligned}
$$

Finally, denote by $\mathscr{F}^{0}$ the smallest $\sigma$-field on $\Omega^{0}$ which makes all marked trees measurable, and let $\mathbf{P}_{x}$ be the probability measure on $\left(\Omega^{0}, \mathscr{F}^{0}\right)$ associated with $X, X_{0}=x$.

Let $\sigma_{y}^{u}: \Omega^{0, u} \rightarrow[0,+\infty]$ be the first hitting time for the particle $u$ to the point $y$, that is,

$$
\sigma_{y}^{u}:= \begin{cases}\inf \left\{s ; \xi_{s}^{u}\left(\omega^{0}\right)=y\right\}, & \text { if }\{\cdot\} \neq \varnothing, \\ +\infty, & \text { otherwise }\end{cases}
$$

Clearly, $\sigma_{y}^{u}$ is for every $u$ a stopping time with respect to $\mathscr{H}_{:}=\left\{\mathscr{H}_{s}^{u} ; s \geq 0\right\}$. 
Setting

$$
\tau_{y}^{u}:= \begin{cases}\sigma_{y}^{u}, & \text { if } \sigma_{x}^{u}<\infty \text { and } \nexists v<u: \sigma_{x}^{v}<\infty \\ +\infty, & \text { otherwise, }\end{cases}
$$

the family $\tau_{x}=\left\{\tau_{x}^{u}: u \in U\right\}$ becomes a stopping line (cf. [3]) in the sense of:

DEFINITION 1. A stopping line $\tau$ is a family of nonnegative random variables $\tau^{u}: \Omega^{0, u} \rightarrow[0, \infty]$, such that:

(i) $\tau^{u}$ is a stopping time with respect to $\mathscr{H}^{u}$ for every $u \in U$,

(ii) the set $L_{\tau}\left(\omega^{0}\right):=\left\{u \in \omega ; \tau^{u}\left(\omega^{0}\right)<\infty\right\}$ has the line property for every $\omega^{0} \in \Omega^{0}$, that is,

$$
u \in L_{\tau}\left(\omega^{0}\right) \Rightarrow\left(\nexists v<u: v \in L_{\tau}\left(\omega^{0}\right)\right)
$$

REMARK 1. This definition differs slightly from that in [3], page 1197, because in our case $\tau^{u}$ may attain "the value" $+\infty$ and, therefore, $u \in L_{\tau}$ in the case $\tau^{u}=\zeta^{u}$. However, this is of no importance in the present case.

To introduce the first passage process, which is the main topic of this paper, assume that $X_{0}=0$, and for $x \geq 0$ define $L_{x}\left(\omega^{0}\right):=L_{\tau_{x}}\left(\omega^{0}\right)$ and

$$
L_{x+}\left(\omega^{0}\right):=\bigcup_{k=1}^{\infty} \bigcap_{n=k}^{\infty} L_{x+1 / n}\left(\omega^{0}\right) \text {. }
$$

Then, clearly, $L_{0}=L_{0+}=\{0\}$.

Definition 2. The process

$$
Z=\left\{Z_{x}:=\left|L_{x+}\right| ; x \geq 0\right\},
$$

where $|\{\cdot\}|$ denotes the number of elements in $\{\cdot\}$, is called the (right-continuous) first passage process associated with $X, X_{0}=0$. The random times $(n=1,2, \ldots)$

$$
\begin{aligned}
T_{1}\left(\omega^{0}\right) & :=\inf \left\{x>0 ; L_{x}\left(\omega^{0}\right) \neq L_{0}\left(\omega^{0}\right)\right\}, \\
T_{n+1}\left(\omega^{0}\right) & :=\inf \left\{x>T_{n}\left(\omega^{0}\right) ; L_{x}\left(\omega^{0}\right) \neq L_{T_{n}+}\left(\omega^{0}\right)\right\}
\end{aligned}
$$

are called the splitting times of $Z$.

In this note it is shown that $Z$ is a continuous-time Galton-Watson process (or a continuous-time Markov branching process in the terminology of Athreya and Ney [1]) and its creation rate and offspring distribution are computed. Further, in the critical case, we characterize the weak limiting behaviour of a sequence $\left\{Z^{(n)}\right\}$ of processes of the type $Z$. These arise from a sequence $\left\{X^{(n)}\right\}$ of branching Brownian motions, scaled to converge to the so-called superBrownian motion. In particular, if the offspring distribution is independent of $n$ and belongs to the domain of attraction of the $1+\beta$-stable law, $0<\beta \leq 1$, 
then the limiting process obtained from $\left\{Z^{(n)}\right\}$ is a random time change of a spectrally positive $1+\beta / 2$-stable process.

In [10] Neveu considers the first passage problem as introduced above but to the lines $(\lambda t-x, t), t \geq 0$, and for a (supercritical) binary branching Brownian motion (see also [3]). It is seen that Neveu's approach is applicable also in our (sub)critical case. In fact, to make the paper more self-contained, when computing the offspring distribution of $Z$ in the next section, the basic facts in Neveu's approach are also recalled.

2. Characterization of the first passage process. In this section $X$ is a (sub)critical branching Brownian motion with offspring generating function $F(u)=\sum p_{k} u^{k}$. Let $\mathscr{A}(u):=\alpha(F(u)-u)$ denote the infinitesimal generating function $(0<u<1)$. We have the following result.

THEOREM 1. Let $Z$ be the first passage process associated with $X$, as previously introduced. Then $Z$ is a continuous-time Galton-Watson process with creation parameter $\gamma:=\sqrt{2 \alpha}$. Let $\left\{r_{k} ; k=1,2, \ldots\right\}$ denote its offspring distribution and let $G(v)=\sum r_{k} v^{k}$ and $\mathscr{B}(v):=\gamma(G(v)-v), 0<u<1$, denote the generating function and infinitesimal generating function, respectively. Then

$$
\mathscr{B}(v)=2\left(\int_{v}^{1} \mathscr{A}(u) d u\right)^{1 / 2}
$$

or, explicitly,

$$
G(v)=\sqrt{2}\left(\sum p_{k} \frac{1}{k+1}\left(1-v^{k+1}\right)-\frac{1}{2}\left(1-v^{2}\right)\right)^{1 / 2}+v .
$$

Further,

$$
\mathbf{E}_{0}\left(Z_{x}\right)=\exp \left(-\sqrt{1-F^{\prime}(1)} x\right)
$$

and, hence, $Z$ is (sub)critical if and only if $X$ is (sub)critical.

Proof. We verify first that $Z$ has the branching and Markov properties. This is done using the strong Markov property at the stopping line $\tau_{x}$. We recall briefly this concept (see [3]): For $\tau:=\tau_{x}$ introduce the stopped $\sigma$-field in $\Omega^{0}$,

$$
\mathscr{F}_{\tau}^{0}:=\bigvee_{u \in U}\left\{\omega^{0} ; u \notin D_{\tau}\left(\omega^{0}\right)\right\} \cap \mathscr{H}_{\tau^{u}}^{u},
$$

where

$$
D_{\tau}\left(\omega^{0}\right):=\left\{u ; \exists v: v<u, v \in L_{\tau}\left(\omega^{0}\right)\right\}
$$

Then we have

$$
\mathbf{E}_{0}\left(\prod_{u \in L_{x}} f^{u} \circ \theta_{\tau^{u}}^{u} \mid \mathscr{F}_{\tau}^{0}\right)=\prod_{u \in L_{x}} \mathbf{E}_{x}\left(f^{u}\right)
$$


where $f^{u}, 0 \leq f^{u}<1$, is for every $u \in U$ a $\left(\Omega^{0}, \mathscr{F} 0\right)$-measurable function and $\theta_{\tau^{u}}^{u}$ is the shift operator $\theta_{s}^{u}$ evaluated at $s=\tau^{u}$. For the definition of $\theta_{s}^{u}$ : $\Omega^{0, u} \cap\left\{\zeta^{u}>s\right\} \rightarrow \Omega^{0}$, see [3]. Informally, $\theta_{s}^{u}$ maps a marked tree $\omega^{0}$ to the marked tree $\tilde{\omega}^{0}$, which is the subtree of $\omega^{0}$ having the particle $u$ at the age $s$ as the first element.

Consider now (2.2) with $f^{u}\left(\omega^{0}\right):=f\left(\omega^{0}\right):=s^{Z_{y+x}\left(\omega^{0}\right)}$, where $0<s<1$ and $x, y \geq 0$. Then it is easily seen that

$$
\begin{aligned}
\mathbf{E}_{0}\left(s^{\left.Z_{y+x} \mid Z_{x}\right)}\right. & =\mathbf{E}_{0}\left(\prod_{u \in L_{x}} s^{Z_{y+x} \circ \theta_{\tau^{u}}^{u} \mid Z_{x}}\right) \\
& =\left(\mathbf{E}_{x}\left(s^{Z_{y+x}}\right)\right)^{Z_{x}} \\
& =\left(\mathbf{E}_{0}\left(s^{Z_{y+x} \mid Z_{x}}=1\right)\right)^{Z_{x}},
\end{aligned}
$$

which is the branching property of $Z$ (cf. [3] Corollary 2.3). A similar computation combined with the spatial homogeneity of $X$ gives the Markov property of $Z$. Consequently, taking into account the fact that the paths of $Z$ are step functions, $Z$ is a continuous-time Galton-Watson process (see [1], page 102). In particular, $Z$ has the properties:

(i) $T_{n}-T_{n-1}, n=1,2, \ldots\left(T_{0}=0\right)$, are independent and, given $Z_{T_{n-1}}=k$, exponentially distributed with parameter $\gamma k$ for some $\gamma>0$.

(ii) $Z_{T_{n}}-Z_{T_{n-1}}, n=1,2, \ldots$ are i.i.d.

To compute the parameter $\gamma$ and the offspring distribution, that is, the distribution of $Z_{T_{1}}$, we need (cf. [10]) the following lemma. Unfortunately, we do not have an exact reference for this, but see Williams ([12], Theorems 4.7 and 4.9). In any case, the lemma can be proved using the reflection principle.

Lemma 1. Let $B=\left\{B_{t}: t \geq 0\right\}, B_{0}=0$, be a standard Brownian motion and $\tau \sim \exp (\alpha)$ independent of $B$. Then the random variables

$$
M:=\sup _{t \leq \tau} B_{t} \quad \text { and } \quad R:=M-B_{\tau}
$$

are independent and exponentially distributed with the parameter $\sqrt{2 \alpha}$. Moreover, the time point for the occurrence of $M$ is a.s. unique.

Let $M^{0}:=\sup \left\{\xi_{s}^{0} ; 0 \leq s<\zeta^{0}\right\}$. Then, by Lemma $1, M^{0} \sim \exp (\sqrt{2 \alpha})$ and, from the definition of $T_{1}, T_{1}=M^{0}$. Hence, $\gamma=\sqrt{2 \alpha}$.

To proceed with the offspring distribution, it follows from the spatial homogeneity and the branching property of $X$ that, in law,

$$
Z_{T_{1}}= \begin{cases}Z_{R^{0}}^{(1)}+\cdots+Z_{R^{0}}^{\left(\nu^{0}\right)}, & \nu^{0} \geq 1, \\ 0, & \nu^{0}=0,\end{cases}
$$

where $Z^{(i)}$ are independent copies of $Z$ evaluated at the independent exponen- 
tial time $R^{0}:=M^{0}-\xi_{\xi^{0}-}^{0}$. Further, recall (see [1] page 106 or Neveu [10]):

Lemma 2. Let $N=\left\{N_{t} ; t \geq 0\right\}, N_{0}=1$, be a continuous-time GaltonWatson process with the creation parameter $\beta$ and offspring distribution $\left\{q_{k}\right.$; $k=0,1, \ldots\}$. For $0<\psi_{0}<1$, let $\psi(t):=\mathbf{E}\left(\psi_{0}^{N_{t}}\right)$, where $\mathbf{E}$ denotes the expectation operator associated with $N$. Then $\psi$ is the solution of the initial value problem

$$
\begin{aligned}
\psi^{\prime} & =\mathscr{G} \circ \psi, \\
\psi(0) & =\psi_{0},
\end{aligned}
$$

where $\mathscr{G}$ is the infinitesimal generating function of $N$, that is,

$$
\mathscr{G}(u)=\beta\left(\sum q_{k} u^{k}-u\right) .
$$

Moreover, in the (sub)critical case, $\psi$ is increasing with $\lim _{t \rightarrow \infty} \psi(t)=1$ and, hence,

$$
\mathscr{G}(u)=\psi^{\prime}\left(\psi^{-1}(u)\right) .
$$

By (2.3), for $0<v<1$,

$$
\begin{aligned}
G(v) & =\mathbf{E}_{0}\left(v^{Z_{T_{1}}}\right)=\mathbf{E}_{0}\left(v^{Z_{R^{0}}^{(1)}+\cdots+Z_{R^{0}}^{(0)}}\right) \\
& =\int_{0}^{\infty} \gamma e^{-\gamma s} \sum p_{k}\left(\mathbf{E}_{0}\left(v^{Z_{s}}\right)\right)^{k} d s .
\end{aligned}
$$

Let $\phi(t):=\mathbf{E}_{0}\left(\phi_{0}^{Z_{t}}\right)$, where $\phi_{0}, 0<\phi_{0}<1$, is given, and $\bar{\phi}(t, v):=\mathbf{E}_{0}\left(v^{Z_{t}}\right)$, when $\phi$ is considered as a function of two variables. Making use of the semigroup property $\phi(t+s)=\bar{\phi}(t, \phi(s))$ and Lemma 2 it is seen that

$$
\begin{aligned}
\phi^{\prime}(t) & =\mathscr{B}(\phi(t))=\gamma\left(\int_{0}^{\infty} \gamma e^{-\gamma s} F(\bar{\phi}(s, \phi(t))) d s-\phi(t)\right) \\
& =\gamma\left(\int_{0}^{\infty} \gamma e^{-\gamma s} F(\phi(s+t)) d s-\phi(t)\right) .
\end{aligned}
$$

Differentiating with respect to $t$, using $(d / d t) F(\phi(s+t))=(d / d s) F(\phi(s+t))$ and integrating by parts,

$$
\begin{aligned}
\phi^{\prime \prime}(t) & =\gamma\left(\int_{0}^{\infty} \gamma e^{-\gamma s} \frac{d}{d s} F(\phi(t+s)) d s-\phi^{\prime}(t)\right) \\
& =-\gamma^{2}(F(\phi(t))-\phi(t))=-2 \mathscr{A}(\phi(t)) .
\end{aligned}
$$

Further, since $\phi^{\prime}(+\infty)=0$,

$$
\phi^{\prime}(t)^{2}=-2 \int_{t}^{+\infty} \phi^{\prime}(s) \phi^{\prime \prime}(s) d s=4 \int_{t}^{\infty} \phi^{\prime}(s) \mathscr{A}(\phi(s)) d s .
$$

By Lemma $2, t \mapsto \phi(t)$ is increasing and therefore, because $\phi(+\infty)=1$,

$$
\phi^{\prime}(t)=\gamma\left(2 \int_{\phi(t)}^{1}(F(u)-u) d u\right)^{1 / 2},
$$


which gives the basic relationship (2.1). Now observe that

$$
G^{\prime}(1)=1-\sqrt{1-F^{\prime}(1)} .
$$

Note also that $G^{\prime \prime}(1)=+\infty$. From this the remaining statements are easily obtained and the proof of the theorem is complete.

REMARK 2. More generally, let $Z_{x}(0, t), x>0, t>0$, be the number of first hits in a family tree to the level $x$ during the time interval $(0, t)$. To convince the reader that the process $Z$ is the "natural" first passage process we point out that in the critical case one can prove

$$
\mathbf{E}_{0}\left(Z_{x}(0, t)\right)=\mathbf{P}_{0}^{B}\left(\tau_{x}<t\right),
$$

where $\mathbf{P}^{B}$ is the measure associated with a standard one-dimensional Brownian motion and $\tau_{x}$ is the first hitting time of the point $x$. This should be compared with the relation

$$
\mathbf{E}_{0}\left(N_{t}(x, y)\right)=\mathbf{P}_{0}^{B}\left(B_{t} \in(x, y)\right)
$$

for $N_{t}(x, y)$ the number of particles in the interval $(x, y)$ at time $t$. Furthermore, there is an analogue of Theorem 1 for supercritical branching. We intend to study these topics in a forthcoming paper.

EXAMPLE 1. Consider the family of critical offspring distributions for $X$ given by

$$
\mathscr{A}(u)=\frac{\alpha}{1+\beta}(1-u)^{1+\beta}, \quad 0<\beta \leq 1 .
$$

For this particular family the offspring distributions can be given explicitly. With $\beta=1$ this is the binary branching model $p_{0}=p_{2}=1 / 2$. For $0<\beta<1$,

$$
\begin{aligned}
& p_{k}=\frac{1}{1+\beta}\left(\begin{array}{c}
1+\beta \\
k
\end{array}\right)(-1)^{k}, \quad k \neq 1, \\
& p_{1}=0 .
\end{aligned}
$$

Note that the variance is finite only for $\beta=1$. The offspring distribution of $Z$ is now of the same type with the infinitesimal generating function

$$
\mathscr{B}(v)=\frac{\gamma}{\sqrt{(1+\beta)(1+\beta / 2)}}(1-v)^{1+\beta / 2}
$$

and offspring probabilities

$$
\begin{aligned}
& r_{k}=\frac{1}{\sqrt{(1+\beta)(1+\beta / 2)}}\left(\begin{array}{c}
1+\beta / 2 \\
k
\end{array}\right)(-1)^{k}, \quad k \neq 1, \\
& r_{1}=1-\sqrt{\left(\frac{1+\beta / 2}{1+\beta}\right)} .
\end{aligned}
$$


Here, $r_{0}$ is the probability that the whole tree lies on the left-hand side of the line determined by the rightmost maximum of the initial particle until the first branching. Furthermore, for $0<\phi_{0}<1$,

$$
\phi(x):=\mathbf{E}_{0}\left(\phi_{0}^{Z_{t}}\right)=1-\frac{1-\phi_{0}}{(1+c x)^{2 / \beta}}, \quad c:=\frac{\gamma \beta\left(1-\phi_{0}\right)^{\beta / 2}}{\sqrt{(1+\beta)(1+\beta / 2)}}
$$

From this formula, setting $\phi_{0}=0$ we obtain the probability $\mathbf{P}\left(Z_{x}=0\right)$ that the whole tree lies on the left-hand side of the line $(x, t), t \geq 0$ (cf. Walsh [11], Proposition 8.14, which has a misprint).

3. Diffusion approximation, critical case. In this section we restrict attention to the critical case and consider a sequence of branching Brownian motions with the interpretation that each particle is assigned a decreasingly small mass and life length, whereas this is balanced by an increasing density of particles. More exactly, we consider a scaling which in a weak limit leads to the distribution of "mass" on the real line known as super-Brownian motion. We characterize the continuous state branching processes (CB processes) obtained in this limit for the associated sequence of first passage processes.

For each $n \geq 1$, let $X^{(n)}$ be a critical branching Brownian motion with creation rate $\alpha_{n}$, generating function $F_{n}$ and infinitesimal generating function $\mathscr{A}_{n}(u)=\alpha_{n}\left(F_{n}(u)-u\right)$. We assume that the processes $X^{(n)}$ all start with one particle at the origin. Let $\left\{X^{(i, n)} ; 1 \leq i \leq n\right\}$ be independent copies of $X^{(n)}$ and let $Z^{(n)}$ and $Z^{(i, n)}$ be the corresponding first passage processes. According to Theorem 1 the characteristics of $Z^{(n)}$ are given by $\sqrt{2 \alpha_{n}}, G_{n}$ and $\mathscr{B}_{n}$, say. Introduce the processes

$$
Y^{(n)}=\left\{Y_{t}^{(n)}=\frac{1}{n} \sum_{i=1}^{n} Z_{t}^{(i, n)} ; t \geq 0\right\}, \quad n=1,2, \ldots
$$

It is well known that a possible limiting process of $Y^{(n)}$ as $n \rightarrow \infty$ can be expressed in terms of a random time change of a spectrally positive Lévy process. This is due to Lamperti [8] for discrete time processes and it was pointed out by Helland that the result also applies to the continuous-time branching processes. Indeed, to see this relation let

$$
H_{n}(v):=G_{n}\left(e^{-v / n}\right) e^{v / n}
$$

and denote by $A^{(n)}, A_{0}^{(n)}=1$, a compound Poisson process with

$$
\log \mathbf{E}\left[\exp \left(-v\left(A_{t+s}^{(n)}-A_{s}^{(n)}\right)\right)\right]=\sqrt{2 \alpha_{n}} n\left(H_{n}(v)-1\right) t .
$$

Then, $Y^{(n)}$ converges in finite-dimensional distributions to a CB process $Y$, $Y_{0}=1$ if and only if $A^{(n)}$ converges weakly to a spectrally positive Lévy process $A, A_{0}=1$.

Further, if the process $Y$ does not explode (for a definition of this see the proof below), then $Y^{(n)}$ converges also weakly to $Y$. See Helland ([6], Theorem 6.1). (The quoted result refers to a slightly different but equivalent setting emphasizing the scaling $t \mapsto n t$; cf. Remark 4.) 
In order to study the sequence $\left\{Y^{(n)}\right\}$ via the relation (3.1) we recall some results for the sequence $\left\{X^{(n)}\right\}$. For $u \geq 0$, let

$$
\mathscr{A}_{*}(u):=c u^{2}+\int_{0}^{\infty}\left(e^{-s u}-1+s u\right) \nu(d s),
$$

where $c \geq 0$ and $\nu$ is a measure on $\mathbf{R}_{+}$such that

$$
\int_{0}^{\infty}\left(s \wedge s^{2}\right) \nu(d s)<\infty .
$$

For each $m>0$ and $n \geq m$ put

$$
\varepsilon_{n}(m):=\sup _{u \leq m}\left|n \mathscr{A}_{n}\left(1-\frac{u}{n}\right)-\mathscr{A}_{*}(u)\right| .
$$

We say that the branching mechanism of $X^{(n)}$ is in the domain of attraction of a branching exponent $\mathscr{A}_{*}(u)$ if

$$
\lim _{n \rightarrow \infty} \varepsilon_{n}(m)=0, \text { for all } m>0 .
$$

Under this assumption it is known that

$$
\frac{1}{n} \sum_{i=1}^{n} X^{(i, n)} \Rightarrow \text { super-Brownian motion with branching exponent } \mathscr{A}_{*}
$$

[weak convergence, e.g., in $\mathscr{D}([0,+\infty), \mathscr{M})$, where $\mathscr{M}$ is the set of finite measures on R]. See Ethier and Kurtz ([5], Section 9.4). In particular, if $N^{(n)}$ $\left(N^{(i, n)}\right)$ denotes the number of particles in $X^{(n)}\left(X^{(i, n)}\right)$, then $N^{(n)}, n \geq 1$, is a sequence of continuous-time Galton-Watson processes such that the total mass process has a weak limit

$$
\frac{1}{n} \sum_{i=1}^{n} N^{(i, n)} \Rightarrow N, \quad t \geq 0
$$

with $h(t):=-\log \mathbf{E}\left[e^{-\theta N_{t}}\right]$ the unique positive solution of

$$
h^{\prime}(t)=-\mathscr{A}_{*}(h(t)), \quad h(0)=\theta .
$$

We now present an analogous result for the first passage process $Z$.

THEOREM 2. Assume that the branching mechanism of $X^{(n)}$ is in the domain of attraction of a branching exponent $\mathscr{A}_{*}$ as in (3.3). Then the sequence of processes $Y^{(n)}, n=1,2, \ldots$, converges weakly to a $C B$ process $Y$, such that its cumulant generating function $h(t):=-\log \mathbf{E}\left[e^{-\theta Y_{t}}\right]$ is the unique positive solution of the equation

$$
h^{\prime}(t)=-\mathscr{B}_{*}(h(t)), \quad h(0)=\dot{\theta},
$$

where

$$
\mathscr{B}_{*}(v)=2\left(\int_{0}^{v} \mathscr{A}_{*}(u) d u\right)^{1 / 2}
$$


Equivalently, $Y$ is a random time change of a spectrally positive Lévy process $A, A_{0}=1$, with the Laplace transform given by

$$
\log \mathbf{E}\left[\exp \left(-v\left(A_{t+s}-A_{s}\right)\right)\right]=\mathscr{B}_{*}(v) t, \quad v \geq 0 .
$$

Proof. We first establish that $Y^{(n)}$ converges in distribution to a process $Y$ which is defined as a random time change of a spectrally positive Lévy process $A$ characterized via (3.5). More specifically, let $\tau_{t}$ be the right continuous inverse of the additive functional

$$
\alpha_{t}:=\int_{0}^{t \wedge T} \frac{d s}{A_{s}}, . \quad T:=\inf \left\{t: A_{t}=0\right\},
$$

and set $Y_{t}:=A_{\tau_{t}}$ in the case $\tau_{t}<T$. Because $A$ is spectrally positive, $A_{t}>0$ for $t<T$ and $A_{T-}=0$. Further, note that if $T=\infty$ and $\alpha_{\infty}<\infty$, then $\tau_{t}$ is not defined for $t>\alpha_{\infty}$. In this case we say that $Y$ has exploded. If $T<\infty$ and $\alpha_{T}<\infty$, we set $Y_{t}=0$ for $t \geq \alpha_{T}$, and say that $Y$ has become extinct.

Now by Theorem 6.1 in [6], it is enough to show that the sequence $\left\{A^{(n)}\right\}$, where $A^{(n)}$ is as in (3.1), converges in finite-dimensional distributions to the process $A$. Since all processes have stationary independent increments, this is equivalent to the convergence in one-dimensional distribution, that is,

$$
\log \mathbf{E}\left[\exp \left(-v\left(A_{t+s}^{(n)}-A_{s}^{(n)}\right)\right)\right]=\sqrt{2 \alpha_{n}} n\left(H_{n}(v)-1\right) t \rightarrow \mathscr{B}_{*}(v) t, \quad n \rightarrow \infty .
$$

However,

$$
\sqrt{2 \alpha_{n}} n\left(H_{n}(v)-1\right)=n e^{v / n} \mathscr{B}_{n}\left(e^{-v / n}\right),
$$

so the desired relation follows from

$$
\lim _{n \rightarrow \infty}\left|n \mathscr{B}_{n}\left(1-\frac{v}{n}\right)-\mathscr{B}_{*}(v)\right|=0 .
$$

But for each $n \geq 1$,

$$
\mathscr{B}_{n}(v)=2\left(\int_{v}^{1} \mathscr{A}_{n}(u) d u\right)^{1 / 2}
$$

according to Theorem 1 . Hence, for fixed $v$ and $n>v$,

$$
\begin{aligned}
\left|n \mathscr{B}_{n}\left(1-\frac{v}{n}\right)-\mathscr{B}_{*}(v)\right| & =2\left|n\left(\int_{1-v / n}^{1} \mathscr{A}_{n}(u) d u\right)^{1 / 2}-\left(\int_{0}^{v} \mathscr{A}_{*}(u) d u\right)^{1 / 2}\right| \\
& =2\left|\left(\int_{0}^{v} n \mathscr{A}_{n}\left(1-\frac{u}{n}\right) d u\right)^{1 / 2}-\left(\int_{0}^{v} \mathscr{A}_{*}(u) d u\right)^{1 / 2}\right| \\
& \leq 2\left(\int_{0}^{v}\left|n \mathscr{A}_{n}\left(1-\frac{u}{n}\right)-\mathscr{A}_{*}(u)\right| d u\right)^{1 / 2} \\
& \leq 2\left(v \varepsilon_{n}(v)\right)^{1 / 2} \rightarrow 0, \quad n \rightarrow \infty,
\end{aligned}
$$

where the assumption (3.2) is used at the last step. 
We now verify that $Y$ does not explode. For this it is enough to show that $T<\infty$ a.s., that is, the process $A$ hits zero a.s. A result due to Zolotarev says that

$$
\mathbf{E}[\exp (-\theta T)]=\exp \left(-\mathscr{B}_{*}^{-1}(\theta)\right), \quad \theta \geq 0,
$$

where $\mathscr{B}_{*}^{-1}$ is the inverse of the continuous and increasing function $\mathscr{B}_{*}$ (for a nice proof, see Bingham [2]). Hence

$$
\mathbf{P}(T<\infty)=\exp \left(-\mathscr{B}_{*}^{-1}(0)\right)=1 .
$$

Finally, because $Y$ is a random time change of a Lévy process, it is strong Markov and has cadlag sample paths. Consequently, its cumulant generating function is the unique solution of (3.4); see Kawazu and Watanabe [7]. The proof is complete.

We next consider some examples of offspring distributions.

EXAMPLE 2. Suppose that the variance $\sigma^{2}$ of the offspring distribution is finite. Take $\alpha_{n}=\alpha n$ and $F_{n}=F$. Then

$$
n \mathscr{A}_{n}\left(1-\frac{u}{n}\right)=n^{2} \alpha\left(F\left(1-\frac{u}{n}\right)-\left(1-\frac{u}{n}\right)\right) \approx \frac{\alpha \sigma^{2} u^{2}}{2}+o\left(\frac{1}{n}\right) ;
$$

hence $\nu(d s) \equiv 0$ and $\mathscr{A}_{*}(u)=\alpha \sigma^{2} u^{2} / 2$. The basic case is binary branching with $F(u)-u=(1-u)^{2} / 2$. We obtain $\mathscr{B}_{*}(v)=\sqrt{(2 \alpha / 3)} \sigma v^{3 / 2}$. Thus $Y$ is a $\mathrm{CB}$ process of index $3 / 2$. The corresponding Lévy process is spectrally positive $3 / 2$-stable.

EXAMPLE 3. A standard example to illustrate the effect of infinite variance is the case

$$
c=0, \quad \nu(d s)=\beta \Gamma(1-\beta)^{-1} s^{-(2+\beta)} d s, \quad 0<\beta<1,
$$

in (3.2). Then

$$
\mathscr{A}_{*}(u)=\frac{\alpha}{1+\beta} u^{1+\beta}, \quad 0<\beta<1 .
$$

If a sequence $\left(\alpha_{n}, F_{n}\right)$ is in the domain of attraction of this stable $1+\beta$ type exponent in the sense of (3.3), then for the first passage process

$$
-\log \mathbf{E}\left[e^{-\theta Y_{t}}\right]=\frac{\theta}{\left(1+\gamma d_{\beta}(\beta / 2) \theta^{\beta / 2} t\right)^{2 / \beta}}, \quad \theta>0, \gamma=\sqrt{2 \alpha},
$$

and

$$
\log \mathbf{E}\left[\exp \left(-v\left(A_{t+s}-A_{s}\right)\right)\right]=\gamma d_{\beta} v^{1+\beta / 2} t, \quad d_{\beta}^{-1}=\sqrt{(1+\beta)(1+\beta / 2)} .
$$

Here, moments of order less than $1+\beta$ are finite. Note that Example 1 with

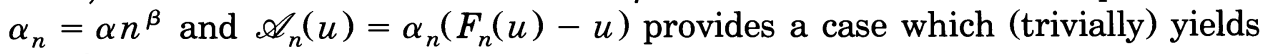
this limit. 
REMARK 3. Suppose that the branching Brownian motions are such that the limiting branching law has the exponent

$$
\mathscr{A}_{*}(u)=\frac{\alpha}{1+\beta} u^{1+\beta}, \quad 0<\beta \leq 1 .
$$

The case $\beta=1$ is Example 2 with $\sigma=1$, and $0<\beta<1$ corresponds to Example 3. Let $a_{n} \sim\left[n^{\beta / 2}\right]$. Then for fixed $\alpha$,

$$
\frac{1}{a_{n}} \sum_{i=1}^{a_{n}} Z_{n t}^{(i)} \Rightarrow Y_{t}
$$

Still another equivalent scaling for $\alpha$ independent of $n$ is

$$
\frac{1}{n} \sum_{i=1}^{n} Z_{n^{\beta / 2} t}^{(i)} \Rightarrow Y_{t}
$$

EXAMPLE 4. To obtain the general form of $\mathscr{A}_{*}$ in (3.2) define, for a given branching exponent $\mathscr{A}_{*}, \alpha_{n}=\mathscr{A}_{*}^{\prime}(n)$ and $F_{n}(u)=u+\mathscr{A}_{*}(n(1-u)) / n \alpha_{n}$. Then $\mathscr{A}_{n}(u)=\alpha_{n}\left(F_{n}(u)-u\right)$ defines an approximating branching mechanism such that (3.2) holds. See Dawson and Perkins ([4], Lemma 3.4c).

EXAMPLE 5. Consider again the situation in (3.6). The extinction probability for the total mass process $N$ is given by

$$
-\log \mathbf{P}\left(N_{t}=0\right)=\lim _{\theta \rightarrow \infty} \frac{\theta}{\left(1+\alpha \beta(1+\beta)^{-1} \theta^{\beta} t\right)^{1 / \beta}}=((1+\beta) / \alpha \beta t)^{1 / \beta} .
$$

Similarly,

$$
-\log \mathbf{P}\left(Y_{t}=0\right)=\left(2 / \gamma \beta d_{\beta} t\right)^{2 / \beta}, \quad \gamma=\sqrt{2 \alpha}
$$

where $d_{\beta}, 0<\beta<1$, was given in Example 3 and $d_{1}=1 / \sqrt{3}$. For example, if $\beta=1$, the asymptotic relation

$$
t \mathbf{P}\left(N_{t}>0\right) \sim 2 / \alpha, \quad t \rightarrow \infty,
$$

has the counterpart

$$
t^{2} \mathbf{P}\left(Y_{t}>0\right) \sim 12 / \gamma^{2}, \quad t \rightarrow \infty,
$$

which gives a quadratic rate of extinction for the number of particles reaching far out to the right.

Acknowledgment. P. Salminen thanks B. Chauvin for a stimulating discussion on the topic of the paper.

\section{REFERENCES}

[1] Athreya, K. B. and Ney, P. E. (1972). Branching Processes. Springer, Berlin.

[2] Bingham, N. H. (1975). Fluctuation theory in continuous time. Adv. in Appl. Probab. 7 705-766. 
[3] Chauvin, B. (1991). Product martingales and stopping lines for branching Brownian motion. Ann. Probab. 19 1195-1205.

[4] Dawson, D. A. and Perkins, E. A. (1991). Historical Processes. Mem. Amer. Math. Soc. 93 (454) 1-179.

[5] Ethier, S. N. and Kurtz, T. F. (1986). Markov Processes: Characterization and Convergence. Wiley, New York.

[6] Helland, I. S. (1978). Continuity of a class of random time transformations. Stochastic Processes Appl. 7 79-100.

[7] KawaZU, K. and Watanabe, S. (1971). Branching processes with immigration and related limit theorems. Theory Probab. Appl. 16 36-54.

[8] Lamperti, J. (1967). Continuous State branching processes. Bull. Amer. Math. Soc. 73 $382-386$.

[9] Neveu, J. (1986). Arbres et processus de Galton-Watson. Ann. Inst. H. Poincaré 22 199-207.

[10] Neveu, J. (1987). Multiplicative martingales for spatial branching processes. In Seminar in Stochastic Processes (E. Çinlar, K. L. Chung and R. K. Getoor, eds.) 223-242. Birkhäuser, Boston.

[11] WaLsh, J. B. (1986). An introduction to stochastic partial differential equations. Ecole d'Eté de Probabilités de Saint-Flour XIV. Lecture Notes in Math. 1180 265-439. Springer, Berlin.

[12] Williams, D. (1974). Path decompositions and continuity of local time for one-dimensional diffusions. Proc. London Math. Soc. 28 738-768.

UPPSALA UNIVERSITET

Matematiska InSTITUTIONEN

THUNBERGSVÄGEN 3

S-752 38 UPPSALA

SWEDEN
Åbo AKADEmI

Matematiska Institutionen

FÄNRIKSGATAN 3 B

SF-20500 А̊во

FinLAND 